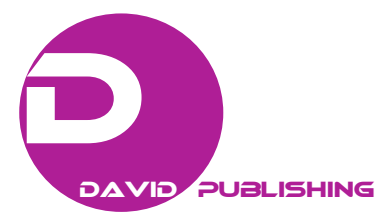

\title{
Acute Pancreatitis with Duodenal Diverticulum
}

\author{
Hamri Asma, Narjis Youssef and Benelkhaiat Ridouan \\ Department of Surgery, Ibn Tofail Hospital, Mohamed VI University Hospital, University of Medicine and Pharmacy, Marrakech \\ 40000, Morocco
}

\begin{abstract}
Intraluminal duodenal diverticulum is a recognised but rare cause of acute pancreatitis. CASE REPORT: we recently encountered this lesion in a 65 year old woman. She had no history of gallstones with no any medications or alcohol consomation. She presented to our hospital because of worsening abdominal pain for three days. The pain was initially in the upper abdomen but later radiated to the back. Epigastric tenderness was evident on physical examination. She had a history of similar painful episodes occurring infrequently for a few years but the pain had never been so severe. She had never previously sought medical attention for this pain. Her pancreatic enzymes were found to be elevated. Interestingly, a subsequent CT scan showed an irregular pancreatic outline consistent with pancreatitis and parapapillary diverticulum $(15 \mathrm{~mm})$. No intervention was needed since the patient's condition started to improve with conservative management and the patient was found to be doing well at his one year follow-up visit with medical treatment. The majority of duodenal diverticula are asymptomatic. Clinical presentation may be characterized by non-specific abdominal symptoms. However, complications are responsible for presentation in most cases.
\end{abstract}

Key words: Pancreatitis, periampullary duodenal diverticulum.

\section{Background}

Pancreatitis in the elderly is a problem of increasing occurrence and is associated with severe complications. Periampullary diverticula are extraluminal outpouchings of the duodenum rarely associated with pancreatitis [1]. We present a case of duodenal diverticulum presenting with pancreatitis to emphasize the importance of this commonly neglected etiology.

\section{Case Report}

We recently encountered this lesion in a 65 year old woman. She had no history of gallstones with no any medications or alcohol consomation. She presented to our hospital because of worsening abdominal pain for three days. The pain was initially in the upper abdomen but later radiated to the back. Epigastric tenderness was evident on physical examination. She had a history of similar painful episodes occurring infrequently for a few years but the pain had never been so severe. She had never previously sought medical attention for this

Corresponding author: Benelkhaiat Ridouan, Ph.D., research field: surgery. pain. Her pancreatic enzymes were found to be elevated. Interestingly, a subsequent CT scan showed an irregular pancreatic outline consistent with pancreatitis and parapapillary diverticulum $(15 \mathrm{~m})$. No intervention was needed since the patient's condition started to improve with conservative management and the patient has had no further symptoms in the 12 months since the medical treatment.

\section{Discussion}

Duodenal diverticulum is a well known entity since the early eighteenth century when it was first reported by a French pathologist, Chomel, in 1710. The duodenum is the second most common site of diverticula in the small bowel following the jejunum [2]. Intraluminal duodenal diverticulum is thought to be part of the spectrum of congenital abnormalities, which includes duodenal atresia [3]. It usually presents in adults, after enlargement of the diverticulum with food or ingested foreign bodies, resulting in obstruction of the duodenal lumen, or more rarely of the ampulla of Vater. Compression of the common bile duct, dysfunction of the ampulla or a poorly emptying 
diverticulum with a narrow neck can all lead to pancreatico-biliary disease and possible pancreatitis or other complications [4], pancreatitis which resolved spontaneously. To date, there are no hard and fast guidelines regarding management of such diverticulum. Earlier this century, surgical diverticulectomy was frequently carried out for non-specific symptoms. There is now consensus that elective surgical treatment of asymptomatic or minimally symptomatic diverticulum is not justified. Surgical or endoscopic interventions should only be reserved for symptomatic diverticulum. Diverticulectomy for vague pain and abdominal discomfort is dangerous and unrewarding; it carries a high morbidity and mortality [5].

\section{Conclusion}

The presence of periampullary diverticula should be excluded before diagnosing idiopathic pancreatitis, particularly in the elderly. However, when a duodenal diverticulum is found in the absence of any additional pathology, only then should the symptoms be attributed to the diverticulum.

\section{References}

[1] Trna, J., Penka, I., Buliková, B., Zbořil, V., and Novotný, I. 2016. "Juxtapapillary Duodenal Diverticulum Causing Pancreatobiliary Problems-Case Report and Literature Review." Rozhl Chir. Summer 95 (7): 294-7.

[2] Chambenois, E., Derhy, S., and Arrivé, L. 2015. "Intraluminal Duodenal Diverticulum. A Rare Cause of Recurrent Acute Pancreatitis." Clin. Res. Hepatol. Gastroenterol. 39 (3): 278-9.

[3] Reichert, M. C., Bittenbring, J. T., Fries, P., Zimmer, V., Lammert, F., and Dauer, M. 2012. "A Case Report: Recurrent Pancreatitis Caused by a Hugeintraluminal Duodenal Diverticulum." J Gastrointestin Liver Dis. 21 (2): 126.

[4] King, A., Peters, C. J., and Shorvon, P. 2012. "Acute Pancreatitis with Pancreatic Abscess Secondary to Sealed Jejunal Diverticular Perforation.” BMJ Case Rep. 2012: bcr1120115255.

[5] Rizwan, M. M., Singh, H., Chandar, V., Zulfiqar, M., and Singh, V. 2011. "Duodenal Diverticulum and Associated Pancreatitis: Case Report with Brief Review of Literature." World J. Gastrointest. Endosc. 3: 62-3. 